\title{
Studienteilnehmer gesucht
}

— Für den Morbus Meniére existiert zwar eine Fülle von Behandlungsstrategien, jedoch gibt es bisher keine nach „state-ofthe-art“-Prinzipien durchgeführten Studien. Frühere Untersuchungen konnten zeigen, dass eine höhere Dosierung von Betahistin therapeutisch wirksamer ist als eine niedrigere. Daher wird nun eine multizentrische, placebokontrollierte, doppelblinde Dosisfindungsstudie durchgeführt.

Ziel ist es, den Effekt von unterschiedlich hoch dosiertem Betahistin auf die Anzahl der Schwindelattacken zu prüfen. Untersucht werden soll dabei insbesondere eine höhere Dosierung von Betahistin $3 \times 48 \mathrm{mg}$ pro Tag), die bisher noch nicht zur Behandlung des Morbus Menière zugelassen ist. Die Studie (EudraCT-Nr. 2005-000752-32) wird vom Bundesministerium für Bildung und Forschung finanziert (BMBF177zfyGT).

Es beteiligen sich zehn Zentren in den folgenden Städten: Aachen, Berlin, ErlangenNürnberg, Essen, München, Regensburg, Tübingen.

Einschlusskriterien für die Studie: Definitiver M. Menière gemäß den Kriterien der
American Academy of Otolaryngology Head and Neck Surgery:

—zwei oder mehr Drehschwindelattacken von mindestens 20 Minuten Dauer

_ Tinnitus oder Völlegefühl im betroffenen Ohr

- Ausschluss anderer Ursachen

- audiometrisch dokumentierter Hörverlust

— mindestens zwei Menière-Attacken pro Monat für mindestens drei aufeinander folgende Monate

_Alter: 18-8o Jahre

Ausschlusskriterien:

— andere Erkrankungen des vestibulären Systems (z. B. vestibuläre Migräne oder phobischer Schwankschwindel)

_ Kontraindikationen für die Behandlung mit Betahistin (z. B. Asthma, Phäochromozytom)

_ Schwangerschaft oder Stillzeit

- schwere Nieren- oder Leberinsuffizienz, Magen- oder Dünndarmulzera, Tumoren, schwere koronare Herzerkrankung

- Behandlung mit anderen Antihistaminika
Behandlungsdauer: neun Monate, weitere drei Monate Follow-up

Klinische Ziele: Reduktion von Schwindelattacken und Tinnitus, Verbesserung der Hör- und Gleichgewichtsfunktion.

Für diese klinisch wichtige Behandlungsstudie werden dringend geeignete Patienten/innen gesucht. Kollegen/-innen, die in Frage kommende Patienten behandeln, können sich direkt bei Herrn Prof. Dr. Michael Strupp über die Studie und das für Sie nächstgelegene Studienzentrum informieren. Dies gilt auch für interessierte Patienten.

\section{Ansprechpartner:}

Prof. Dr. Michael Strupp

Klinikum derLudwig-Maximlians-Universität München,

\section{Campus Großhadern}

Neurologische Klinik und IFB Schwindel

Tel: 089 / $7095-6678$ oder 6680

Fax: 089 / 7095-6673

E-Mail: Michael.Strupp@med.uni-muen-

chen.de

www.schwindelambulanz-muenchen.de

\section{Keine Nullrunde für Ärzte}

\section{Köhler rechnet mit Honorarplus im nächsten Jahr}

- Trotz des Rekorddefizits der gesetzlichen Krankenkassen im kommenden Jahr beharrt die Kassenärztliche Bundesvereinigung (KBV) auf ihrer Forderung nach einem Honorarzuwachs für die rund 140.000 Vertragsärzte in Deutschland. In den bevorstehenden Verhandlungen im Bewertungsausschuss aus Ärzten und Kassen gehe es aus Sicht der KBV „ausschließlich“ um die Frage, „ob und in welchem Umfang“ es Zuwächse beim Honorar gebe, sagte KBV-Chef Dr. Andreas Köhler in Berlin. Seinem Wissensstand nach plane die Koalition keine Nullrunde für die Ärzte. „Wir gehen nach wie vor davon aus, dass es nur Zuwachsbegrenzungen geben wird.“

Köhler verwies in diesem Zusammenhang auf ein Sparpapier der Unionsfraktion. Da- nach soll der vom Bewertungsausschuss festzulegende Anstieg bei der Honorarsumme 2011 auf 50 Prozent begrenzt werden. Auf diese Weise sollen rund 500 Millionen Euro eingespart werden.

Einsparungen im Gesundheitswesen dürften aber nicht allein auf das „Kollektivvertragssystem abgewälzt“ werden, so Köhler. Auch Kliniken, Apotheken oder Hausarztverträge müssten ins Visier genommen werden. Köhler schlug zudem eine Neuregelung der Praxisgebühr vor. Diese müsse von derzeit zehn auf fünf Euro gesenkt, dafür aber bei jedem Arztbesuch fällig werden. Köhler begründete dies mit der hohen Zahl an Arztbesuchen. Mit im Schnitt 18 Arztkontakten jährlich seien Deutschlands Patienten international Spitzenreiter.

hom

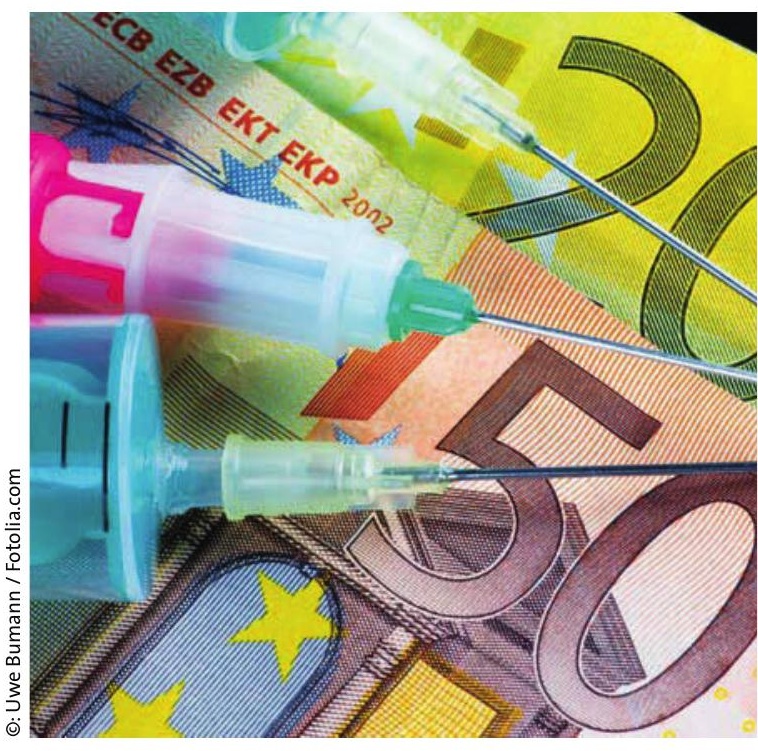

